\title{
Du « matheux » bourgeois au « Quant " corporate
}

\author{
Les héritiers des classes supérieures \\ scientifiques à l'épreuve de la finance \\ londonienne
}

\begin{abstract}
Marlène Bouvet ${ }^{1}$
[Résumé] Comment des héritiers des classes supérieures scientifiques, animés par l'amour désintéressé des mathématiques, se muent-ils en agents financiers acquis à l'esprit « corporate» ? Cet article propose l'examen minutieux des métamorphoses du «capital culturel scientifique » des Quants, ingénieurs-mathématiciens à la City de Londres. Une enquête de six mois, élaborée à partir de neuf entretiens semi-dirigés et d'un corpus de lettres de motivation et grilles de salaire, met au jour la fabrique des dispositions contradictoires qui façonnent leur ethos professionnel. Porteurs de puissantes dispositions ascétique et scholastique, chevillées à une appétence forcenée pour les sciences, ils sont cependant tenus d'acquérir des manières d'être inédites. Disqualifiant un élitisme fondé sur la distinction intellectuelle, la culture d'entreprise sollicite une disposition distante, une disposition rationnelle-stratégique et une disposition vénale qui font violence au « sérieux ludique » présidant jusque-là à l'exercice de leurs facultés. Ils s'initient alors, non sans peine, à des compétences pédagogique et mondaine qui rompent avec leur habitus de domination par l'excellence scolaire, et tentent littéralement de mettre à profit leurs compétences techniques. Si elle dépend de la durée d'exposition à la culture d'entreprise, l'attitude des Quants face à l'adversité s'avère fortement contrastée : leurs adaptations différenciées réfractent le capital culturel hétérogène des diverses fractions de la bourgeoisie française. Si la stylisation des dispositions professionnelles enrichit la sociologie d'un groupe professionnel, celui des agents financiers, elle permet également de capturer les récentes transformations de la culture des élites.
\end{abstract}

Mots-clés : dispositions; finance ; bourgeoisie ; ingénieur ; élites ; capital culturel scientifique.

[Abstract] How can inheritors of the upper scientific classes, driven by a disinterested, scholarly taste for mathematics, turn into financial agents committed to the corporate spirit? This paper offers a detailed analysis of the socialization process that shape their "scientific cultural capital", as they learn to domesticate math with the family, at school, and into the financial world. Drawing on semi-structured interviews and professional archives (a collection of cover letters and pay scales), it unveils the making of the conflicting dispositions that make up the professional ethos of French "Quants", who work as mathematicians-engineers in the City of London. Driven by powerful ascetic and

${ }^{1}$ École normale supérieure de Lyon. 
scholastic dispositions, combined with a relentless craving for science, Quants nevertheless have to incorporate new relational social skills. More than intellectual prowess, corporate culture calls for a disposition to emotional detachment, a rational-strategic disposition, and a venal disposition that runs against the playful seriousness that Quants cultivated before entering the labour market. To convert their technical mastery into economic capital, Quants have to develop pedagogical and social skills which break with a habitus built on academic superiority. Confronted with these challenges, Quants display a wide range of attitudes. While these uneven adaptive strategies certainly reflect seniority, some variations mirror subtle differences that can be found in terms of cultural capital between distinct fractions of the French scientific bourgeoisie. The stylized depiction of professional dispositions captures both the unsung practices of an occupational group and the latest transformations of elite culture.

Keywords: disposition; finance; bourgeoisie; engineer; elite; scientific cultural capital.

Le 21 août 2013, Les Échos recensaient une anecdote des plus spectaculaires sur le monde de la finance : un stagiaire allemand, Moritz Erhardt, décédait après 72 heures de travail acharné au sein de la Bank of America Merrill Lynch. Bien que l'étudiant soit décrit comme une « victime de la culture managériale de la City à l'égard de ses jeunes recrues » (Roche, 2013), il apparaît en filigrane que son identification à l'exigeant modèle d'autoréalisation proposé par la banque préexistait à son intégration du poste. Ainsi, déclarait-il, dans l'une de ses ultimes publications Facebook: «Je viens d'une famille qui favorise l'excellence, qui vit sous pression, d'où mon tempérament ambitieux et très compétitif ». S’y dessine la stratification d'une socialisation familiale et scolaire ayant conditionné l'hyper-investissement de ce jeune homme au sein de la banque. Si la culture d'entreprise catalyse ce tragique évènement, les injonctions de l'organisation semblent surtout solliciter une disposition ascétique ancienne, jusqu'à son point de rupture.

L'outillage d'une épistémologie dispositionnaliste s'impose dans l'édification d'une sociologie des Quants, parce qu'elle prend à revers leur écrasante invisibilisation sur le plan des représentations. Dépouillés de toute épaisseur sociale, ils représenteraient en effet ce que la finance contemporaine a de plus abstrait et désincarné : de simples cerveaux mis au service d'une dynamique d'auto-perfectionnement des marchés financiers. Or l'existence même de l'ingénieur-mathématicien impose de réviser l'imagerie mythique du "golden-boy». Ingénieurs en Recherche et Développement, ces acteurs « d'arrière-plan » œuvrent, en étroite collaboration avec le trader, à la réduction des risques. Ils établissent des modèles mathématiques de prévision des prix fondés sur des formules de probabilité extrêmement complexes. Pour prendre des décisions d'achat, le trader ne s'en remet donc plus à son seul flair : il intègre les recommandations du modèle, encadré par une équipe de professionnels supérieurement qualifiés.

L'apparition de cette profession dans l'organisation du travail financier découle d'une transformation historique. Jusqu'à la fin du vingtième siècle, le bourgeois amateur de transactions boursières déléguait la réalisation de celles-ci à des professionnels du marché, agents de change formant une corporation hiérarchisée (Godechot, 2001). 
Cependant, la dérégulation impulsée dans les années quatre-vingt entraîne une diminution graduelle de «l'esprit maison », tandis que l'article de Black et Scholes suscite un boom du marché des produits dérivés ${ }^{2}$. La soudaine mathématisation des marchés induit une sensible augmentation du niveau de diplôme des intervenants, dont la figure par excellence est l'ingénieur quantitativiste. Titulaire d'une thèse en physique ou mathématiques, ou du « passeport pour la finance » que constitue le Master El Karaoui il détient généralement la palme des titres scolaires dans la salle de marché. Dans la division du travail financier, la profession de Quant participe d'une tension à l'œuvre entre deux pôles : le « Centre de profit » (Front-office), où s'affairent les traders en charge de leurs portefeuilles, et le "Centre de coût »(Middle-office), considéré comme un pesant impératif structurel. À ces deux pôles, s'ajoute le Back-Office, rouage ignoré de l'organisation ; ses employés se chargent des aspects techniques du règlement des transactions. Au sein d'un univers où « la proximité au profit fait la noblesse » (Godechot et Blancard, 2008 : 212), la profession de Quant tend à être assimilée au «Centre de coût ». Cependant, l'enquête révèle que ses compétences s'actualisent en différents points du spectre ${ }^{4}$.

Les Quants constituent donc, par excellence, les agents de la nouvelle culture scientifique qui soutient le processus de rationalisation amorcé en Occident quatre siècles auparavant (Weber, 2010 [1904-1905]). Comment dresser le patrimoine dispositionnel qui caractérise ces héritiers des classes supérieures scientifiques, enfants de la bourgeoisie poursuivant le rêve de la grande richesse ? Comment décrire au mieux, sur les plans épistémologique et méthodologique, la métamorphose d'un capital culturel scientifique hérité en un "capital technologique » mâtiné de compétences relationnelles ? Enfin, comment rendre compte, au sein d'un univers qui se veut strictement intéressé et pragmatique, de la concurrence entre formes d'élitisme marchand et intellectuel, et des conséquences de cette hybridation sur l'exercice concret de la profession?

C'est la rencontre d'Andrea, ambitieuse « chasseuse de tête » sicilienne de vingt-sept ans, qui catalysa l'enquête conduite de janvier à mai 2013, par entretiens semi-dirigés,

\footnotetext{
2 Paru en 1973, il propose un instrument d'évaluation des prix des options : une «simple » formule mathématique. Le calcul stochastique permet, en prenant en compte l'évolution du cours du produit sous-jacent, d'évaluer ce prix avec une justesse inédite.

${ }^{3}$ Le Master El Karaoui, créé en 1990 à l'Université Pierre-et-Marie Curie, est le master de mathématiques financières le plus réputé de Paris. "Master Pro » des élites scientifiques scolaires désireuses d'intégrer la finance, il produirait des éléments fin prêts à l'immersion dans la banque.

${ }^{4}$ Le « Model-Validation Quant » détecte les déficiences des instruments financiers au sein du Middle-office. Garde-fou obligé, il joue un rôle d'audit interne, modérant les ardeurs des aventuriers téméraires qu'incarnent traders et vendeurs. Le Front-Office Quant, cependant, répond aux besoins les plus pressants du Trader en matière de cadrage de l'information, tandis que le Quant-Trader fait du trading à haute fréquence et gère des subordonnés.
} 
auprès de neuf Quants. Un stage auprès de cette recruteuse en freelance m'a fourni une prise complémentaire sur cet univers relativement clos, via des grilles de salaire détaillées, des fiches de poste et un corpus de lettres de motivation de soixante-deux Polytechniciens désireux d'accomplir leur stage dans la finances. La plupart de mes enquêtés travaillent dans les banques leaders du capitalisme mondial (Société Générale, BNP Paribas ou Bank of America Merrill Lunch). L'immixtion dans une fête en appartement, un breakfast dans un pub branché ou la consommation de bières au pied de la City m'ont permis d'observer leurs compétences relationnelles et leur rapport pratique aux "plaisirs de Londres ». Quoique désirable sur le plan scientifique, une ethnographie de plain-pied dans la salle de marché aurait appelé à d'intenses négociations; dans un contexte où la seule sollicitation d'un entretien enregistré éveillait la suspicion des Quants Senior, j’y renonçaí.

\section{Le capital culturel " scientifique " des ingénieurs financier : un patrimoine hybride de dispositions, compétences et savoir- faire}

Plutôt que d'accréditer un individualisme méthodologique susceptible de consacrer une lecture " coût/avantage » des comportements, il convient d'adopter une épistémologie qui dénaturalise l'action financière. Appréhender le marché, c'est en effet conquérir un objet sociologique longtemps « considéré comme le sanctuaire des sciences économiques - et nul sociologue, anthropologue ou historien ne pouvait avoir l'audace de se l'approprier» (François, 2008: 6). Angle mort d'un prisme saisissant la banque comme échelle d'analyse privilégiée 7 , l'appréhension des agents qui la font fonctionner concrètement s'avère pourtant pleinement légitime. Rendre à l'activité financière sa profondeur historique et individuelle implique d'illuminer la fabrique sociale des agents qui s'y dédient, comme le fait magistralement Bourdieu (1977) lorsqu'il historicise la "propension au calcul» des paysans kabyles. À l'image des entrepreneurs algériens, dont certains s'approprient les outils de l'économie capitaliste (la monnaie, l'intérêt) tandis que d'autres échouent à les conquérir, les Quants présentent un degré d'incorporation différencié des schèmes de perception et d'action qu'induit l'adaptation à la banque. C'est précisément la mise au jour de résistances qui met à mal «l'anthropologie imaginaire » des économistes (Bourdieu, 2017).

\footnotetext{
${ }^{5}$ Précipité des valeurs cardinales que promeut le champ économique (dynamisme et innovation), ces lettres renseignent également sur les titres et hauts faits des prétendants au poste de Quant.

${ }^{6}$ Voir l'annexe «Les Quants: caractéristiques sociales, statut dans la banque et "style de vie" ».

${ }^{7}$ Les économistes orthodoxes considèrent la banque comme une entité homogène maximisant son profit, et la résument à son rôle d'intermédiation financière dans le fonctionnement de l'économie (Freixas et Rochet, 1997).
} 
Qu'engage la mobilisation du paradigme dispositionnaliste, du point de vue du type singulier de capital que déploient les Quants dans leur quotidien professionnel ? Dans un contexte « d'accélération technologique du monde [...], il aurait été remarquable que la description que Bourdieu fait du capital culturel [...], canon traditionnel d'humanités orienté vers la culture légitime [...], continue d'exister sous une forme inchangée » (Prieur et Savage, 2013 : 261). Si l'on définit plus largement le capital culturel comme un « ensemble de pratiques éducatives » visant la réussite scolaire et sociale, on conçoit aisément qu'« il existe toujours une culture légitime et dominante, mais [...] que les compétences qui fonctionnent comme du capital culturel évoluent» (Draelants, 2014). Il faut donc, pour saisir les inégalités corrélatives à létat actuel du monde social, capturer et définir de nouvelles formes de capitaux. La mise au jour d'un capital singulier ne doit cependant rien sacrifier à la rigueur ; il s'agit d'éviter l'écueil de «l'infinie scissiparité » du concept de capital, pour le rattacher aux «trois espèces fondamentales » (économique, culturel et social) que consacre la trinité bourdieusienne (Neveu, 2013). Puisque toute disposition relève du capital culturel incorporé, l'enjeu décisif consiste à déceler - et fixer théoriquement - le contenu du capital culturel en mutation qui équipe cette bourgeoisie bancaire.

Énucléer la logique secrète des savoir-faire à l'œuvre dans l'exercice d'une telle profession requiert, selon nous, d'explorer l'intuition de Bourdieu sur l'avènement d'un « capital technologique $»^{8}$. Désireux de la formaliser, Archer et al. (2015) trouvent dans son principe un "capital scientifique » qu'ils conceptualisent comme la combinaison entre « les différents types de capitaux économique, social et culturel qui auraient spécifiquement trait à la science »-sans que le capital scientifique puisse être considéré «comme un type séparé de capital». Le "capital scientifique » procède donc d'une forme de capital culturel familial particulier, qui fluctue en fonction de la fraction de la bourgeoisie afférente à la formation morale des Quants. À l'intérieur', le capital culturel scientifique recouvre des facultés, « habitudes d'esprit » impliquant « la capacité au raisonnement déductif, la capacité à identifier des variables pertinentes, des schémas dans les données, une familiarité avec le calcul numérique et une disposition d'esprit critique ». Il inclut d'autre part une « culture scientifique » (science literacy) relevant plutôt de « la compréhension de la façon de [...] se servir de ces facultés dans la vie quotidienne pour le bénéfice personnel et social» (Claussen \& Osbone, 2013 : 68). Or, c'est précisément l'usage intéressé de ces facultés qui parachève l'acquisition des « dispositions [...] pertinentes dans l'exercice » du métier de Quant, et le respect des « règles non écrites de son art » (Zarca, 2009).

\footnotetext{
${ }^{8}$ « Un portefeuille de savoirs scientifiques [...] ou de ressources techniques (procédures, routines, aptitudes, savoir-faire unique et cohérent, capables de réduire le coût du travail et du capital) [...], déployé dans la conception et la manufacture de produits " (Bourdieu, 2005 :194, cité dans Archer et al., 2015 : 927).

${ }^{9}$ Les deuxième et troisième volets du capital scientifique incluent des pratiques de consommation en rapport avec les sciences, et des formes de capital social en rapport avec la science (Archer et al. : 929).
} 
Pour autant, les ressorts concrets de l'exploitation du capital culturel scientifique par les classes supérieures demeurent excessivement flous, notamment en situation professionnelle. Nous distinguerons donc trois niveaux d'intelligibilité sociologique des schèmes de comportement qui forment ce capital : (1) la disposition, schème permanent, durable et transversal à plusieurs contextes d'action, souvent chargé d'une dimension éthique ; (2) la compétence, capacité à mobiliser différentes ressources (savoirs, savoir-faire, savoir-être) en vue de résoudre efficacement une situation complexe (Parlier et Gilbert, 1992). C'est « une potentialité et pas une inclination relativement permanente », qui s'actualise dans des contextes limités (Lahire, 2002 : 415) ; (3) les savoirs, séries de pratiques observables qui découlent de l'exercice des compétences dans des contextes d'action encore plus bornés. S'agissant de l'exécution d'un exercice de mathématiques, «savoir résoudre une équation du second degré » constitue une compétence, une « habitude d'esprit » propres au capital scientifique. Elle se manifeste concrètement dans le savoir-faire que constitue « la résolution pratique de l'équation par le suivi d'une procédure standardisée » (Lahire, 2002 : 415).

Remonter jusqu'aux dispositions nécessite de repenser cet enchâssement à partir de chaque pratique observable. En effet, plusieurs dispositions peuvent concourir à l'exercice d'une même compétence. Par exemple, l'ascétisme et la disposition scolastique sont des dispositions qui, conjuguées, conduisent l'ingénieur à exercer ses compétences en mathématiques avec d'autant plus d'ardeur qu'il éprouve une appétence pour les savoir-faire impliqués : ces dispositions l'inclinent à ériger la virtuosité du modèle comme un objectif en soi. En revanche, un patrimoine dispositionnel aux antipodes - conjugaison du calcul stratégique, de la vénalité, des compétences mondaines et du détachement - incitera le Quant à mobiliser ses compétences relationnelles et sa « culture scientifique » pour présenter ses modèles (même médiocres) comme particulièrement lucratifs auprès des interlocuteurs idoines.

En somme, on ne peut penser une série de dispositions professionnelles sans s'exposer au risque d'une contradiction dans les termes. Si les manières de faire et de sentir récurrentes sont propres au seul contexte professionnel, elles sont savoir-faire ou compétences. Il faut donc concevoir la transmission, la fabrique et l'actualisation des compétences en même temps que celles des « dispositions professionnelles ». Si, par un mécanisme endogène cumulatif, la détention du capital culturel scientifique tend à faciliter son accumulation ultérieure (Archer et al., 2015), il importe d'étudier finement les continuités derrière les ruptures apparentes entre les facultés convoquées par les cours de mathématiques en contexte scolaire et les prouesses techniques exigées en contexte professionnel (« faire une addition », « élaborer un modèle financier »). 


\section{La genèse du patrimoine dispositionnel d'héritiers des classes supérieures scientifiques}

Dans ces familles de la bourgeoisie française scientifique, l'école et la famille «fonctionnent, inséparablement, [...] comme des marchés qui, par leurs sanctions positives ou négatives [...], renforcent ce qui est “acceptable”, [...] vouant au dépérissement les dispositions dépourvues de valeur» (Bourdieu, 1979: 93). Puisque la discipline reine des mathématiques fonctionne comme un vaccin d'immunité scolaire, elle sera surinvestie par les enfants : «En gros voilà, quelqu'un de bon en maths, même s'il a des mauvaises notes par ailleurs, il va jamais être critiqué... » (Damien, Model-Validation Quant de 30 ans). Soulignons d'abord la forte prévalence des classes supérieures scientifiques parmi les parents des ingénieurs mathématiciens : pour cinq d'entre eux, l'un des parents (souvent les deux) est ingénieur.e, médecin, pharmacien.ne ou professeure de physique et mathématiques. L'on observe, au cours d'une scolarité très encadrée dans le secteur privé, une forte incitation parentale au développement de cette compétence en mathématiques - y compris sur un mode distant, insensible et ludique (Ferrand, Imber et Marry, 1999: 56).

Cette part de capital hérité est perceptible dans la similitude des compétences parentales et enfantines : alors que la mère de Blanche (Quant Junior de 26 ans) est professeure de physique, quatre de ses cinq enfants s'engagent dans des études d'ingénieur. En outre, la majorité des enquêtés témoignent de réticences précoces au "français » (dès l'école primaire), par opposition à l'instantanéité de la compréhension en mathématiques : «J'ai toujours été assez mauvais en français. [Pourquoi ?] Parce que, parce que j'étais pas doué pour ça, j'en sais rien. [...] C’est pas un problème de pas me plaire, c'est que... que j’y arrive pas ! *signes d'agacement* [...] J'ai plus de mal avec le par cœur aussi. J'ai un esprit qui est plus synthétique. » (Jacques, 30 ans, Front-Office Quant, parents médecins). S’agissant des mécanismes de socialisation qui président à la transmission de ces capitaux culturels, l'anecdote de Frédéric (Senior, 43 ans) illustre à merveille la « manière douce » par laquelle le goût des mathématiques peut être transmis : « Je crois que ça a été déclenché - j’ai eu une crise d'appendicite, j’avais neuf ans, et j'étais à l'hôpital, à Madagascar. [...] Et en fait, pour tromper la faim, ma mère, qui avait toujours un bon esprit scientifique, elle m’a fait résoudre une équation - équation du premier degré, équation à une inconnue... Et en fait donc, j’ai très bien résolu [...]. Parce que c'était un jeu ! C'était pas du tout dans un contexte... scolaire». L'acquisition de l'« esprit mathématique » (expression de Frédéric) apparaît ainsi comme le résultat d'habitudes éducatives et d'une transmission de capitaux culturels scientifiques. Elle relève en outre d'un éthos masculin fondé sur la «force neuronale » (Castelain-Meunier, 1988), la prévalence de cette compétence parmi les hommes s'expliquant largement par « les attentes différenciées des maîtres dans les matières supposées convenir inégalement aux élèves des deux sexes » (Duru-Bellat, 1995 : 87). 
Enfin, la préférence parentale pour les mathématiques peut se manifester sur un mode quasi injonctif, au détriment d'autres appétences. À l'âge de huit ans, les intérêts de Damien, dont le père ingénieur assure le train de vie dans le $16^{e}$ arrondissement de Paris, se portent d'abord sur les mots : « J'aimais bien la... la littérature [...] j'écrivais, ben... plus ou moins des, des petits romans, enfin... [Sur quoi ?] Ben des, des histoires qui pouvaient m'arriver, des trucs imaginaires, de la poésie [...]. J’avais écrit aussi un livre en fait, sur un inspecteur ou un truc comme ça... ». Mais il sera rapidement "recadré » dans ses ambitions ; lorsqu'il s'agit d'opérer des choix d'orientation, il s'approprie subjectivement son destin de " garçon » dans la famille : « En gros c'est assez conservateur, le garçon lui, il doit... il doit réussir à avoir un bon métier, et les filles en fait, elles font un peu ce qu'elles veulent ». Damien se persuade qu'il préfère les mathématiques à la littérature, qui acquiert le statut d'amour de jeunesse. De même, alors que Blanche fait " quatre heures par jour » de danse classique jusqu'en Terminale, elle fait le choix d'intégrer une prestigieuse école d'ingénieurs pour éviter la précarité de ses amies danseuses de ballet "obligées de travailler à mi-temps chez Franprix », et ainsi accéder à une « belle situation ». Ces compétences en mathématiques, produites par l' « idéologie charismatique du don » comme une forme de supériorité intellectuelle native (Bourdieu et Passeron, 1964), préparent bien évidemment leur mutation en personnel financier hautement qualifié.

C'est également le cas de la disposition ascétique, mobilisée en vertu d'un solide sens de la compétition et d'une habitude des classements : « La rationalisation d'activités physiques répétitives et organisées, le régime de vie extrêmement strict, l'exercice scolaire "illimité", [...] révèlent [...] une éthique de l'effort et de l'autocontrainte» (Darmon, 2013 : 276). L'aisance avec laquelle six sur neuf de mes enquêtés font l'expérience de la "prépa " scientifique, et la récurrence de performances sportives exceptionnelles (quatre sur neuf) en témoignent: ce passage à travers une institution qui "porte presque violemment les esprits au travail » (Durkheim, 1990 : 295-300) demeure, à une exception près, un « bon souvenir ».

L'enquête révèle cependant qu'aux deux pôles de la stratification sociale, correspondent des types d'ascétisme qui concourent presque dans les mêmes proportions à la formation de dispositions professionnelles "opératoires ». Dans son enfance, Medhi, issu de milieu populaire, s'inscrit dans une logique apparemment similaire ; un « ordre moral domestique » strict semble faire office de substitut aux capitaux culturels dans ses succès scolaires (Lahire, 1995). En effet, il grandit parmi les adultes d'un bourg algérien ; en tant qu'aîné de sept sœurs, il doit se montrer à la hauteur des espoirs paternels. Pour se rendre à l'école, il lui faut traverser une forêt sur deux kilomètres, « avec tout [son] barda sur le dos ». Son père contrôle toutes les dimensions de son existence : « Il était très consciencieux, mon père, hein ! Il était toujours derrière ses enfants, à vérifier les devoirs même s'il avait été que deux ans à l'école, ça faisait un peu policier. [...] Il disait: "Celui-là il est pas bien, tu traînes pas avec lui !" ». Il développe ainsi des habitudes que l'on peut qualifier d'ascétiques, consacrant tous ses efforts à la mémorisation 
de ses leçons : « J’ai travaillé très dur, depuis tout le temps. » Ses tâches quotidiennes de fils d'agriculteur ne l'empêchent nullement de s'adonner à cet exercice : "Au début, même ce que je comprenais pas je l'apprenais par cœur. Je monte sur mon arbre, y'a les brebis à côté *rires* et j'apprends. [...] J'étais un peu systématique, je fais un programme avec un emploi du temps de tout mon temps libre, de $7 \mathrm{~h}$ à $8 \mathrm{~h}$ je vais manger, etc. J'allais faire tous les exercices du livre ». Mehdi saute des classes et, vers l'âge de 13 ans, gagne le Concours national de physique en Algérie. Sans doute son «engagement » dans le « cercle vertueux de la réussite scolaire » s'explique-t-il par une synergie entre le contrat moral passé avec son père et les recommandations concrètes de son professeur de physique, « aiguilleur scolaire » le « formant et l'informant » de la valeur de ce titre (Castets-Fontaine, 2011 : 6). Ce premier exploit académique aura un effet d'entraînement qui le conduira jusqu'à la soutenance d'un doctorat sur les microparticules. Que révèle ce profil improbable de la disposition ascétique des autres Quants ? Il apparaît que cet « ascétisme populaire [...] souvent lié à l'ambition de mobilité» (Bourdieu, 1979 : 452453), fondé sur « l'acceptation systématique des privations » et «la mobilisation générale des forces pour saisir toutes les occasions de travail »(Schwartz, 2012 [1990] : 110111), se fait sur le mode d'une rudesse systématique, « cas atypique » qui met en lumière, par contraste, l'ascétisme passion (fondé sur l'appétence, l'adhésion, l'enchantement) typique du mode d'investissement des enfants de la bourgeoisie lors de leur socialisation primaire.

Puisque les parents initiés «ne laissent pas les performances ou le simple mérite scolaire de [leurs] enfants décider de leur avenir » (Draelants, 2014 : 21), d'éventuels ratés - passage par l'université, échec en prépa concernant deux Quants - entraînent des processus de «rattrapage », comme le Master El Karaoui. Dans l'ensemble cependant, le rapport de ces "héritiers » à l'école et au travail se caractérise par le "sérieux ludique » distinctif de leur classe d'origine ; pour l'adopter, « il faut être de ceux qui ont pu, sinon faire de leur existence, comme l'artiste, une sorte de jeu d'enfant, du moins prolonger très tard, parfois tout au long de la vie le rapport au monde de l'enfance (tous les enfants commencent leur vie comme des bourgeois, dans un rapport de puissance magique sur les autres et [...] sur le monde, mais ils sortent plus ou moins tôt de l'enfance)» (Bourdieu, 1979: 57). Ainsi, leur distance à la nécessité induit une certaine indifférence initiale à « la signification sociale de l'argent » (Zelizer, 2005); il leur a paru naturel de pratiquer de nombreuses activités extra-scolaires, fréquenter des établissements privés, être entretenus durant leurs études et vivre dans une relative insouciance économique. S'ils choisissent une profession qui leur permet de perpétuer ce rapport au monde propre à la skholè ${ }^{10}$, tout en maintenant un train de vie et une image

\footnotetext{
${ }^{10}$ Tendance à considérer les intérêts de connaissance du champ scientifique comme prioritaires sur les formes de nécessité pratique qui priment dans le monde social. La situation de skholè renvoie à « un temps libre et libéré des urgences du monde, qui rend possible un rapport libre et libéré à ces urgences et au monde » (Bourdieu, 1997 : 9).
} 
de soi conformes à leur statut social originel, l'arrivée dans la banque reconfigure leur rapport aux compétences mathématiques et à l'argent.

\section{Développer une " personnalité business »}

À leur arrivée dans la banque, les Quants se confrontent à un univers professionnel qui épouse bien des aspects de leur socialisation antérieure. Pourtant, il manque à ces champions de l'ordre scolaire des qualités qui s'imposent insensiblement comme impératives : la disposition rationnelle-stratégique, l'appétence pour la communication dans une position subordonnée, et un goût de l'argent résultant du " processus de vénalisation » (Godechot, 2001). Intervient donc un travail d'ajustement qui prendra des années, processus douloureux nécessitant de placer « l’accent de réalité » des mathématiques pures au «monde de l'entreprise et ses exigences propres» (Abraham, 2007: 46). Nos enquêtés se situent cependant à différents stades d'incorporation de cet éthos idéal : elle dépend de leur niveau de séniorité, mais aussi d'une origine sociale qui se réfléchit dans la justesse de leurs pronostics autant que de leurs manœuvres mondaines.

Parmi les dispositions adjuvantes à une bonne incorporation de l'éthos de Quant, l'on compte l'ascétisme, une disposition scolastique soutenant l'absorption dans des opérations de mathématiques abstraites, et un sens de la compétition en " affinités électives » avec le dispositif de la rémunération par le bonus. Sans revenir sur la disposition ascétique, qui se manifeste dans un volume horaire de $60 \mathrm{~h}$ par semaine (Coulangeon, 2011: 198), nous montrons ici comment le capital culturel scientifique accumulé s'actualise dans la manufacture de modèles de prévision des prix.

Jean illustre efficacement l'investissement «désintéressé » du jeu intellectuel qui consiste à les façonner. S'il insiste sur l'importance de l'application pratique des mathématiques, il fait de la dimension d'" aventure intellectuelle » le pilier de son investissement dans la banque. Il s'attarde longuement sur l'histoire des mathématiques financières et convoite le poste de Research Quant, pourtant en voie d'extinction. Professeur de prépa en mathématiques reconverti, Frédéric glose quant à lui sur la symbolique du cercle comme figure de la perfection dans la Grèce antique ; il se fend d'une critique acerbe de l'enseignement bassement calculatoire que délivre l'Éducation Nationale. Enfin, Damien élabore en son for intérieur de véritables traités financiers; il témoigne de certaines compétences de l'universitaire qu'il a choisi de ne pas être, irriguant son travail des dernières trouvailles théoriques présentées dans des articles. Intéressé par le «business » autant que par les mathématiques, il ne sépare jamais l’un de l'autre et tente d'adopter des initiatives innovantes. Il s'agit par exemple de passer d'un modèle déductif à un modèle inductif : « J'suis toujours en train d'apprendre hein ${ }^{*}$ rire* $^{*}[. .$.$] Au fur et à mesure des années en fait, ... j’ai compris que j'faisais plus des$ maths en fait mais de la... de la physique expérimentale. [Ah!Vraiment ? Comment ça ?] [...] Voilà, empiriquement je vais relever en fait tout ce qui est vrai - par exemple en fait, 
si c'est une action qui bouge, comme si c'était un, un insecte, tu vas regarder en fait quels sont ses mouvements [...] et ensuite je vais commencer en fait à... à modéliser ça. ». La dimension de challenge mathématique - les stagiaires se targuent de " penser hors des sentiers battus $»^{11}$ - demeure ainsi pour ces Quants l'un des « motifs personnels d'engagement » dont, selon Chiapello et Boltanski (1999), les jeunes cadres contemporains ont besoin pour accepter la perte de la stabilité professionnelle autrefois au cœur du « second esprit du capitalisme ». En effet, « pour valoir qu'on s'y engage, pour être attrayant, le capitalisme doit pouvoir être présenté à leurs yeux dans des activités qui [...] peuvent être qualifiées d'« excitantes », [...] porteuses de possibilités d'autoréalisation et d'espaces de liberté » (Ibid. : 221). La possibilité de prendre du plaisir dans un domaine fétiche, sans pour autant sacrifier un train de vie bourgeois, fait partie de ces opportunités.

Cependant, l'intégration à la banque suppose également l'adoption d'un certain nombre d'attitudes « implicites » : le calcul stratégique, indissociable d'une faculté à expliquer le fruit de son travail (compétence pédagogique et mondaine), la vénalisation (sensibilité croissante à l'argent comme bien symbolique), l'acceptation d'une position subordonnée au Trader et l'apprentissage du détachement émotionnel. Si toutes apparaissent comme problématiques, nous nous concentrerons ici sur le calcul rationnelstratégique. Sans doute constitue-t-il un marqueur de classe : l'on peut relire l'attitude de prospection scolaire des parents comme une stratégie typique des «minorités choisissantes » du " pôle privé des classes moyennes » (Gombert et Van Zanten, 2004). On pensera également à l'investissement hautement stratégique du temps et des loisirs en classes préparatoires (Darmon, 2003). Néanmoins, le sens tactique que déploie Damien dans ses interactions professionnelles procède à notre sens d'une forme de rationalité proprement économique. Par opposition au principe de non-conscience qui sous-tend les dispositions calculatoires ordinaires des agents (Bourdieu, 1968), il revêt un caractère explicite et délibéré. C'est donc la conscience et la rationalisation à l'œuvre dans le déploiement de ce sens tactique qui motivent notre choix de l'intituler « disposition rationnelle-stratégique ».

Relatant les rivalités qui l'opposent à ses collègues, Damien expose avec clarté les données du problème : «En général, les gens veulent gagner plus d’argent, et puis voilà. Ça, c'est... une constante. Donc ça c'est ce que vous avez en commun. Voilà. Donc après, y'a plusieurs méthodes pour, pour gagner plus d'argent.» Plusieurs méthodes ou plusieurs stratégies, puisqu'il considère ces termes comme synonymes ; il s'agit d'être plus brillant que les autres, meilleur communicant, « flibustier » qui vole les modèles de ses subordonnés... Dans cette course au bonus, qui peut doubler leur salaire annuel, les Quants gagent qu'une aura de compétence favorise une belle prime. Ainsi, les Quants se scinderaient en deux catégories: ceux qui restent dans une logique désintéressée, et finalement aveugle vis-à-vis de leur travail (« intéressé par les modèles

\footnotetext{
${ }^{11}$ L'expression « think out of the box » surgit dans plusieurs lettres de motivation.
} 
pour les modèles, la théorie pour la théorie »), et ceux qui finissent par « voir la réalité des choses » : « Pour comprendre ça, il faut comprendre d'où viennent les Quants, donc ils viennent d'études scientifiques. [...] Et en fait, à partir du moment où... les Juniors deviennent Quants, qui est une porte d'entrée dans le domaine du business, les gens en fait vont apprendre à évoluer... Pas forcément - alors soit ils vont rester... Scientifiques, rester dans des modèles et jamais voir la réalité, soit ensuite, selon la personnalité ben ils vont... vouloir faire du trading... [...] Y'a des personnalités plus business, plus pragmatiques». Aujourd'hui acquis à l'esprit «corporate», Damien manifeste une pointe de condescendance envers ceux qui n'ont pas encore su « faire le chemin » vers le trading; il souhaite intégrer un hedge fund, afin de se confronter à des défis mathématiques plus complexes et plus lucratifs. La dichotomie des profils de Quants s'origine certainement dans des différences de socialisation : le "sérieux des affaires " de Damien tient probablement à son statut d'« héritier » parisien, lycéen « rebelle » initié à la procédure d'achat d'une action vers l'âge de dix ans, par opposition au cas de Jean, lauréat du concours Kangourou encore habité du « souci scolaire » caractérisant les « égarés » d'HEC ${ }^{12}$.

Or pour maximiser son gain, encore faut-il savoir « communiquer » au sujet de son modèle mathématique et de ses mérites, compétence relationnelle difficilement acquise par un grand nombre d'entre eux : «Sur le marché de l'emploi des cadres, [...] ce qui importe [est] d'être capable de présenter sa personnalité sous un jour favorable, [...] en tenant compte des critères de comportement valorisés et évalués dans ce contexte. [...] Le capital culturel institutionnalisé, tout en étant plus que jamais nécessaire [...], est de moins en moins suffisant pour se distinguer » (Draelants et Ballatore, 2017). Mal à l'aise sur le terrain de la présentation de soi, la majorité des Quants n'ont pas de mots assez durs pour la dénoncer. Seule Blanche, Model-Manager qui « fait le lien entre les Quants, les Traders et la model-validation », se voit distinguée pour ses qualités didactiques. Ses employeurs détectent probablement en elle une disposition genrée à la verbalisation (le poste requiert « beaucoup de relationnel »), qui fait défaut aux collègues que Jacques qualifie ironiquement de " geeks » ou d' « Asperger ».

Or faire œuvre de pédagogie, c'est aussi se mettre en valeur pour maximiser le montant de son bonus. Blanche l'analyse avec acuité: "Y'a une bonne partie des Quants qui sont... très forts en technique, mais qui sont pas forcément très forts en communication et du coup ça les gêne pour leur carrière. Parce qu'ils sont pas capables de se vendre, d'expliquer que c'est plus malin que le voisin». Thomas, fils de pharmaciens de province passé par HEC, déplore précisément qu'il y ait dans l'évaluation des employés « un côté un peu mafia »: «Tu vois, c'est pas vraiment ta

\footnotetext{
12 «Au lieu [...] de chercher à s'investir dans la « vie de campus », ils [se préoccupent] de leurs activités scolaires, comme mus par une espèce de nécessité intérieure » (Abraham, 2007: 54). Ce fils d'une ingénieure à l'Éducation Nationale se soucie peu du montant de sa rémunération : «Ma première motivation c'est de continuer d'apprendre ».
} 
performance non plus, c'est... Ché pas, plein d'autres facteurs en fait. [...] C’est très politique, euh..., si le chef il t'aime pas, ben voilà il te vire pour payer ses potes ». Se présentant comme « moyen » en mathématiques, il fait partie de ceux qui « n'ont à faire valoir que leur «foi» dans le «business» et sont contraints [...] de s'efforcer de conquérir par eux-mêmes, en payant de leur personne et par une espèce d'exagération constante, [les] ressources et compétences qui leur font défaut » (Abraham, 2007: 60). Aussi ce « dévot » surinvestit-il le jeu, et tente-t-il de s'initier à cette intelligence des situations spécifiquement relationnelle : « J’lis plus des bouquins de self-improvement, tu vois. Donc j'lis des trucs un peu chiants... Comment être plus corporate, tu vois, plus efficace dans son travail, sur les jeux politiques... ».

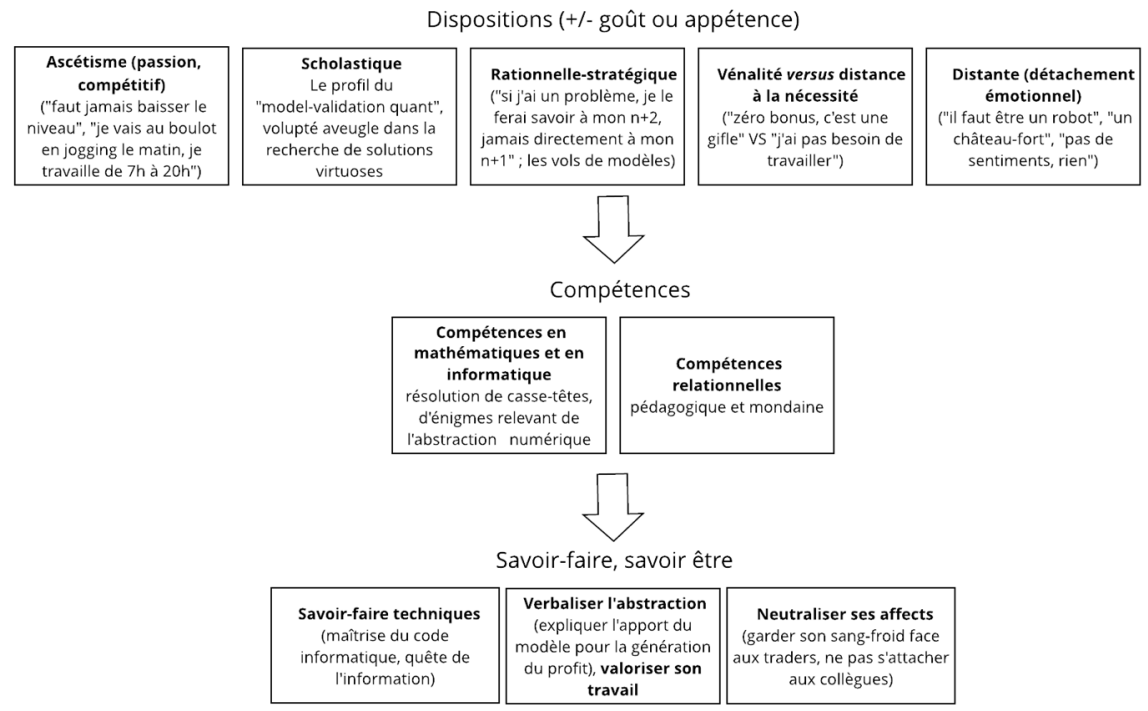

Figure 1. Ethos professionnel du Quant

Moins jaloux de sa légitimité, Damien effectue mille contorsions pour tenir « le chef de son chef » au courant de ses derniers travaux, afin d'éviter l'appropriation de ses modèles par son supérieur hiérarchique direct : «À chaque fois que je suis pas d'accord avec des décisions, des remarques qu'on me fait, je... J'en parle à $n+2$, et puis comme ça voilà, pour régler... les choses... [T’en parles pas à ton supérieur direct.] Non, non. [...] Si y’a une personne en fait, qu'elle crée un conflit et que plusieurs personnes sont en conflit, la personne qui va se faire virer, c'est la personne qui aura raison en fait [...]. Donc c'est - c'est la théorie des jeux ». "Comme un poisson dans l'eau » au sein de l'entreprise (Abraham, 2007: 57), Damien est peut-être le Quant qui objective le plus le fait d'agir de manière calculatrice, sans rien sacrifier de sa disposition scolastique. Un tableau du patrimoine dispositionnel idéal du Quant stylise l'éthos professionnel mixte qu'il incarne (cf. figure 1). 
On sent que Jacques souhaite s'initier à ces jeux, sans nécessairement s'y sentir aussi à l'aise que son cousin : il informe ouvertement la personne qu'il réprouve son geste, sur un ton glacial (" je dis à la personne que c'est inapproprié »), pour se retrouver en début d'année avec un bonus du montant de zéro (" une gifle »)... Chez ce Quant issu de «la Haute », convaincu d'appartenir à la noblesse intellectuelle de la salle de marché, la disposition distante, la disposition scolastique et une certaine hauteur aristocratique compromettent l'actualisation d'une disposition rationnelle-stratégique. Certes, le refus de s'« abaisser » aux jeux de pouvoir permet à Jacques de maintenir son illusio professionnel et un ethos de l'honneur, mais il est également susceptible de fragiliser sa position de dominant. Cependant, au moment de l'entretien, Jacques n'a que cinq ans d'expérience dans la banque à son actif. On peut gager qu'avec la montée en âge et en rémunération, mais aussi à force de sanctions, il fera l'apprentissage d'une capacité à faire profil bas et à rationaliser les « coups par derrière » pour la bonne cause (Vlad).

\section{4. Étudier les dispositions professionnelles pour mettre à nu la culture des élites}

Ainsi, une sociologie dispositionnaliste met en lumière l'effort d'incorporation acharné d'un ethos, mais aussi une forme de préparation culturelle à la conciliation d'injonctions contradictoires au sein de l'entreprise. L'on observe concomitamment l'actualisation de compétences et de dispositions antérieures à la banque - compétence en mathématiques, dispositions ascétique, scolastique, vénale et distante, calcul stratégique de classe - et l'intégration, parfois à marche forcée, de dispositions étrangères à l'illusio et l'habitus bourgeois - rationnelle-stratégique, vénale et au détachement. Si elle donne chair à une sociologie des agents financiers, la stylisation du patrimoine dispositionnel de cette nouvelle bourgeoisie ouvre surtout la conceptualisation aux composantes les plus subtiles du type de capital culturel qui fait mouche dans la compétition professionnelle des présentes élites économiques.

De fait, le souci d'historiciser les comportements des salariés financiers instruit nécessairement les inégalités sociales d'orientation «par le haut» (Draelants, 2014). Notre sociologie des élites informe donc les mécanismes mouvants de la reproduction sociale, éclairant les métamorphoses d'une distinction qui, dans le champ professionnel, s'appuie désormais sur les sciences appliquées, l'argent et la mobilité internationale (Coulangeon, 2011). Il faudrait néanmoins interroger les critères d'une " continuité » des dispositions et des compétences tout au long de la trajectoire sociale et professionnelle des Quants, notamment sur le plan du savoir-être. De surcroit, les difficultés d'adaptation contrastées à l'éthos professionnel de Quant dessinent, en pointillés, une division sociale du travail qui mériterait une investigation plus systématique : Traders masculins issus des Grandes écoles, femmes Quants confinées 
dans le rôle de «Polars » ou de communicatrices, Quants de la bourgeoisie provinciale évincés des positions les plus lucratives ${ }^{13} \ldots$

Exploiter à plein la focale dispositionnaliste requiert enfin d'investiguer la corrélation entre l'augmentation du revenu et le déploiement de diverses tentatives de recomposition du capital culturel, au sens strict (arts nobles) comme sous la forme de loisirs distinctifs dans la vie privée. Si la majorité des Quants se réclament d'une «bonne volonté culturelle » de principe (Bourdieu, 1979), beaucoup admettent consacrer leurs soirées à la lecture d'articles « utilitaires » - l'envahissement du temps libre pouvant être interprété comme l'effet d'une synergie entre dispositions rationnelle-stratégique et scholastique. Pourtant, certains d'entre eux s'initient à l'opéra, tandis que d'autres en profitent pour développer des loisirs "plus coûteux, plus rares » (Coulangeon, 2011 : 142), tels que l'hélicoptère, l'avion ou le ballet, dans la même logique d'élitisme et d'excellence qu'en contexte professionnel. Néanmoins, le cas de Medhi, transfuge adepte $\mathrm{du}$ « moules frites » et tenant d'un langage mixte rompant avec la retenue bourgeoise, suggère que ces pratiques restent le fruit de leurs socialisations successives, que la banque ne saurait définitivement gommer.

\section{Annexe. Les Quants : caractéristiques sociales, statut dans la banque et « style de vie »}

\section{Les juniors : l'artiste et le prétendant}

Blanche : reproduire le mode de vie parental et satisfaire ses dispositions esthétiques

Situation : 25 ans, célibataire.

Type de Quant : model-validation Quant.

Profession du père : directeur général d'une PME commercialisant des verres optiques.

Profession de la mère : professeure de physique-chimie au lycée.

Origine géographique : Courbevoie, banlieue parisienne aisée (92).

Trajectoire scolaire : établissements privés jusqu'au bac. Cours de danse classique en Sport Études au collège et cours de danse privés (4h par jour). À 14 ans, elle passe un an à l'École de Danse de l'opéra de Marseille. École préparatoire scientifique. Intègre SUPELEC (obtient I'ENSAE simultanément). Imperial College de Londres. Master El Karaoui.

Autre (élément de cadrage) : ses trois frères, ainsi qu'elle-même, sont ingénieurs.

Loisirs : ballets et opéras.

Sports (passés et présents) : danse classique intensive.

\footnotetext{
${ }^{13}$ Français aisés expatriés à Londres, les Quants qui ont fait l'objet de cette étude ne constituent en effet qu'une fraction limitée de ce groupe professionnel.
} 
Laurent : l'ascétisme au travail, l'hédonisme dans la dépense

Situation : 26 ans, célibataire.

Type de Quant : Quant-Trader.

Profession du père : commercial en assurances.

Profession de la mère: commerciale en publicité, secrétaire de direction (bac professionnel).

Origine géographique : nombreux déménagements.

Trajectoire scolaire: saute une classe au collège. Classe prépa. École Polytechnique.

Autre (élément de cadrage) : une dizaine de déménagements à travers le monde, au gré des emplois paternels. Son frère a réalisé une thèse de physique.

Loisirs : œnologie, shopping.

Sports (passés et présents) : ski, jogging quotidien.

\section{Les trentenaires de la grande bourgeoisie : le bourgeois décomplexé} et le bourgeois sérieux

Damien : moderniser un mode de vie bourgeois et manœuvrer « rationnellement » pour accéder à la grande richesse

Situation : 30 ans, en couple non cohabitant.

Type de Quant : model-validation Quant.

Profession du père : ingénieur ayant fait l'École Centrale (son grand-père également).

Profession de la mère : femme au foyer.

Origine géographique : $16^{\mathrm{e}}$ arrondissement de Paris.

Trajectoire scolaire: établissements privés catholiques jusqu'au primaire. Entretien d'admission et classement des élèves en 6 e . Bac S. Licence à l'université. Master El Karoui.

Autre (élément de cadrage) : cousin professeur d'université à Polytechnique.

Loisirs : sorties dans des discothèques privées de Soho.

Sports (passés et présents) : inconnu. 
Jacques : se montrer digne de l'héritage parental et financer des loisirs bourgeois

Situation : 30 ans, célibataire.

Type de Quant : front-office Quant.

Profession du père : médecin (le grand-père aurait « fondé une grande marque de savon française », un autre « a fait Sciences Po »).

Profession de la mère : médecin.

Origine géographique : $16^{\mathrm{e}}$ arrondissement de Paris.

Trajectoire scolaire : établissement privé catholique (non-mixte), au sein duquel «les baskets sont interdites». Bac S à 16 ans, école préparatoire scientifique. DEA de probabilités/statistiques à l'université de Jussieu. École d'ingénieur de maths prestigieuse : I'ESCP Grandes Écoles, agrégation, Master El Karoui.

Autre (élément de cadrage) : cousin de Damien, il aurait « de la famille en politique » donc refuse de mentionner le parti pour lequel il vote ; mentionne en entretien qu'il n'a «pas besoin de travailler».

Loisirs : hélicoptère (qui lui a été offert par sa famille).

Sports (passés et présents) : escrime de compétition, tennis de table en club.

\section{Les trentenaires de la petite bourgeoisie provinciale : le savant comblé, le prétendant frustré}

Jean : reproduire le mode de vie parental et construire l'estime de soi dans la disposition scholastique aux mathématiques

Situation : 30 ans, célibataire.

Type de Quant : model-validation Quant.

Profession du père : ingénieur « dans le privé ».

Profession de la mère: ingénieur «au service informatique du rectorat, dans l'administration nationale ».

Origine géographique : inconnue.

Trajectoire scolaire : a gagné le concours Kangourou. Classe préparatoire scientifique. Intègre Centrale Paris.

Autre (élément de cadrage) : néant.

Loisirs : lecture du Canard Enchaîné.

Sports (passés et présents) : tennis de table dans le même club que Jacques. 
Thomas : une tentative frustrée de s'illustrer dans le « business »

Situation : 34 ans, célibataire.

Type de Quant : model-validation Quant (ex Front-office Quant).

Profession du père : pharmacien, docteur en pharmacie.

Profession de la mère: pharmacienne, ancienne professeure agrégée de mathématiques

Origine géographique : petite bourgeoisie alsacienne (Strasbourg).

Trajectoire scolaire : école préparatoire scientifique, HEC, Master El Karaoui.

Autre (élément de cadrage) : un séjour à Tokyo en tant qu'étudiant lui fait prendre conscience de l'importance de bien gagner sa vie. Son objectif de vie principal consiste à acheter une maison à Londres.

Loisirs : inconnu.

Sports (passés et présents) : inconnu.

\section{Les Seniors : dominants financiers et prétendants culturels}

Vlad : « être entrepreneurial » mais convertir une partie des capitaux économiques en capitaux culturels

Situation : 36 ans, célibataire.

Type de Quant : Quant-trader, chef d'équipe (executive director).

Profession du père : médecin.

Profession de la mère : cadre technique à la SNCF moldave (coordination des trains).

Origine géographique : «petit village de Moldavie».

Trajectoire scolaire : concours de mathématiques au lycée ; intégration de l'académie des sciences économiques; mathématiques de haut niveau dans une université de Roumanie.

Autre (élément de cadrage) : son grand-père, entrepreneur agricole, aurait « accueilli l'administration moldave avec un fusil » lors de la collectivisation des terres.

Loisirs : opéra, music-hall.

Sports (passés et présents): champion d'haltérophilie dans son adolescence $\left(3^{\mathrm{e}}\right.$ national). 
Frédéric : I'argent au service de l'affirmation élitiste de soi

Situation : 43 ans, célibataire.

Type de Quant : model-validation Quant, chef d'équipe (executive director).

Profession du père : professeur de lettres classiques.

Profession de la mère : professeur de lettres modernes.

Origine géographique : Madagascar (ex colonie française).

Trajectoire scolaire : école publique, « de très bon niveau » à Madagascar. Agrégation de mathématiques. Ex-professeur de mathématiques en école préparatoire scientifique. Autre (élément de cadrage) : descendants de colons norvégiens à Madagascar, ont joui d'un statut social important (plusieurs propriétés sur lîle, réputation d'un trésor caché) jusqu'à l'indépendance (départ précipité, perte des biens). Propriétaire d'un quatre pièces en face de la City.

Loisirs : avion, échecs (tournois d'échecs internationaux).

Sports (passés et présents) : conduite automobile (Jaguar qu'il possède également).

Mehdi : homo oeconomicus et Quant « poète»

Situation : 47 ans, marié, deux enfants.

Type de Quant : front-office Quant, chef d'équipe (executive director).

Profession du père : éleveur d'ovins (agriculture vivrière).

Profession de la mère : femme au foyer.

Origine géographique : «village oublié de tous», le long du littoral, en Kabylie.

Trajectoire scolaire: II saute plusieurs classes. Premier au concours national de physique en Algérie. Bourse du gouvernement français pour travailler à l'université. Thèse de physique. Post-docs.

Autre (élément de cadrage) : néant.

Loisirs : plaisirs de la table, alcool.

Sports (passés et présents) : inconnu.

\section{Bibliographie}

ABRAHAM Y. (2007), « Du souci scolaire au sérieux managérial, ou comment devenir un "HEC" ", Revue française de sociologie, vol. 48, p. 37-66.

Archer L., Dawson E., Dewitt J., Seakins A., Wong B. (2015), « “Science capital”: A Conceptual, Methodological, and Empirical Argument for Extending Bourdieusian Notions of Capital Beyond the Arts », Journal of Research in Science Teaching, vol. 52, $\mathrm{n}^{\circ}$ 7, p. 922-948. 
Boltanski L., Chiapello E. (1999), Le nouvel esprit du capitalisme, Paris, Gallimard (« NRF essais »).

Bourdieu P., Chamboderon J.-C., Passeron J.-C. (1968), Le métier de sociologue, Paris, Mouton de Gruyter, (« Les textes sociologiques, 1 »).

Bourdieu P. (1977), Algérie 60 : structures économiques et structures temporelles, Paris, Éditions de Minuit («Grands documents»).

Bourdieu P. (1979), La Distinction. Critique sociale du jugement, Paris, Minuit (« Le sens commun »).

Bourdieu P. (1997), Méditations pascaliennes, Paris, Le Seuil (« Liber »).

Bourdieu P. (2017), Anthropologie économique. Cours au Collège de France 1992-1993, Paris, Le Seuil (« Raisons d'agir »).

CASTETS-FonTAINE B. (2011), « La randonnée vertueuse d'élèves de Grandes Écoles issus de "milieux populaires" ", L'orientation scolaire et professionnelle, vol. 40, $\mathrm{n}^{\circ} 1$. En ligne, consulté le 3 juin 2018, URL : https://journals.openedition.org/osp/2971.

CASTElain-Meunier C. (1988), Les hommes d'aujourd'hui, virilité et identité, Paris, Belfond/Acropole («Questions de vie »).

COUlANGeON P. (2011), Les métamorphoses de la distinction. Inégalités culturelles dans la France d'aujourd'hui, Paris, Grasset (" Mondes vécus »).

DARmon M. (2008), Devenir anorexique. Une approche sociologique, Paris, La Découverte (« La Découverte/Poche »).

DARmon M. (2010), « Des jeunesses singulières. Sociologie de l'ascétisme juvénile», Agora débats/jeunesses, $n^{\circ}$ 56, p. 49-62.

DRAELANTS H. (2014), « Des héritiers aux initiés ? Note sur les nouvelles modalités de la reproduction sociale par l'école», Social Science Information/Information sur les sciences sociales, vol. 53, $\mathrm{n}^{\circ}$ 4, p. 403-432.

DraElANTS H., BALLATORE M. (2014), « Capital culturel et reproduction scolaire : un bilan critique ", Revue française de pédagogie. En ligne, consulté le 27 avril 2018. URL : http://journals.openedition.org/rfp/4430.

DuRKheim E. (1990 [1938]), L'évolution pédagogique en France, Paris, Presses universitaires de France ("Quadrige »).

DURU-BELlLAT M. (1994), « Filles et garçons à l'école, approches sociologiques et psychosociales », Revue française de pédagogie, vol. 109, p. 111-141. 
FERRAND M., IMBER F., MARRY C. (1999), L'excellence scolaire : une affaire de famille. Le cas des normaliennes et normaliens scientifiques, Paris, L'Harmattan ("Bibliothèque de l'éducation »).

FreIXAs X., Rocher J.-C. (1997), Microeconomics of Banking, Cambridge, The MIT Press.

Godechot O. (2001), Les traders. Essai de sociologie des marchés financiers, Paris, La Découverte (« Textes à l'appui. Enquête de terrain »).

Godechot (2008), «Les bonus accroissent-ils les risques ?», in P. ARTUS, J.-P. BETHEZE, C. DE Boissieu, G. CAPElle-BlanCARD, La crise des subprimes, Paris, La documentation française, p. 203-218.

LAHIRE B. (1995), Tableaux de familles, Paris, Gallimard (« Le Seuil »).

LAHIRE B. (1998), L'homme pluriel. Les ressorts de l'action, Paris, Nathan («Essais \& recherches. Sciences sociales »).

Gombert P., VAn ZANTEn A. (2004), «Le modèle éducatif du pôle "privé” des classes moyennes: ancrages et traductions dans la banlieue parisienne », Éducation et Sociétés, vol. $14 \mathrm{n}^{\circ}$ 2, p. 67-83.

Gilbert P., PARLIER M. (1992), «La compétence : du mot valise au concept opératoire », Actualité de la formation permanente, $\mathrm{n}^{\circ} 116, \mathrm{p} .11-18$.

Roche M. (2013), « Polémique à la City après la mort d'un stagiaire de Bank of America », Le Monde, publié en ligne le 22 août 2013, consulté le 2 février 2013, URL: https://www.lemonde.fr/economie/article/2013/08/22/polemique-a-la-city-apresla-mort-d-un-stagiaire-de-bank-of-america_3465103_3234.html.

NEVEU E. (2013), « Les sciences sociales doivent-elles accumuler les capitaux ? À propos de Catherine Hakim, Erotic Capital, et de quelques marcottages intempestifs de la notion de capital », Revue française de science politique, vol. 63, n² 2, p. 337-358.

SChwarTz O. (2012 [1990]), Le monde privé des ouvriers, Paris, Presses universitaires de France («Quadrige »).

WEBER M. (2010 [1904-1905]), L'éthique protestante et l'esprit du capitalisme, Pocket/Plon (« Agora »).

ZARCA B. (2009), "L'ethos professionnel des mathématiciens », Revue française de sociologie, vol. $50, \mathrm{n}^{\circ} 2$, p. 351-384.

ZeLLIZER V. (2005), La signification sociale de l’argent, Paris, Le Seuil (« Liber »). 